\title{
Fattori di rischio per tromboembolismo venoso e profilassi nei pazienti ricoverati in Medicina Interna: analisi dallo studio FADOI "GEMINI"
}

\section{Risk factors for venous thromboembolism and prophylaxis in medical inpatients: data from the FADOI "GEMINI" study}

\section{Mauro Campanini ${ }^{a}$, Gualberto Gussoni ${ }^{b, *}$, Mauro Silingardi ${ }^{c}$, Gianluigi Scannapieco $^{\mathrm{d}}$, Carlo Buniolo ${ }^{\mathrm{e}}$, Antonella Valerio ${ }^{\mathrm{b}}$, Walter Ageno ${ }^{\mathrm{f}}$, Ido Iori ${ }^{c}$, Antonino Mazzone ${ }^{g}$, a nome del Gruppo di Studio GEMINI ${ }^{1}$}

\footnotetext{
${ }^{a}$ Dipartimento di Medicina Interna, Ospedale Maggiore della Carità, Novara

b Fondazione FADOI - Dipartimento per la Ricerca Clinica "Centro Studi", Milano

"Medicina Interna I e Stroke Unit, Arcispedale "S. Maria Nuova", Reggio Emilia

'Direzione Sanitaria, Ospedale Ca' Foncello, Treviso

e QBGroup SpA, Padova

${ }^{f}$ Clinica Medica, Università dell'Insubria, Varese

${ }^{\mathrm{g}}$ Dipartimento di Area Medica, Ospedale Civile di Legnano (IMI), Milano
}

Ricevuto il 7 agosto 2009; accettato l'11 settembre 2009

disponibile online il 9 febbraio 2010

\section{KEYWORDS}

Venous

thromboembolism;

Risk factors;

Prophylaxis;

Internal Medicine.

\begin{abstract}
Summary
Background: Though venous thromboembolism (VTE) frequently occurs in non-surgical setting, epidemiology and risk factors for VTE in unselected medical inpatients have not been extensively studied, and uncertainties remain about the prophylactic strategy in these patients.

Materials and methods: In a prospective, observational, multicenter study we aimed to contemporarily assess the epidemiology of symptomatic VTE in consecutive patients hospitalized in Internal Medicine, to evaluate the impact of potential risk factors, and the attitude of internists towards thromboprophylaxis. A total of 4,846 patients were included, during the period March-September 2006.

Results: Symptomatic VTE was registered in 177 (3.65\%) patients; of these, 26 cases $(0.55 \%)$ occurred with onset of symptoms $\geq 48$ hours after admission ("hospital-acquired" events, primary study end-point). Previous VTE and bed resting were significantly associated with venous
\end{abstract}

\footnotetext{
* Corrispondenza: Fondazione FADOI, Dipartimento per la Ricerca Clinica "Centro Studi”, via G.B. Bazzoni 8 - 20123 Milano.

E-mail: gualberto_gussoni@yahoo.it (G. Gussoni).

1 I componenti del gruppo di studio GEMINI sono elencati in Appendice.
} 
thromboembolism, while a trend for increased risk was documented in cancer patients. During hospital stay antithrombotic prophylaxis was globally administered in $41.6 \%$ of patients, and in $58.4 \%$ of those for which prophylaxis was recommended according to 2004 guidelines by the American College of Chest Physicians. The choice of administering tromboprophylaxis appeared qualitatively adherent to indications from randomized trials and international guidelines, and bed rest was the strongest determinant of the use of prophylaxis.

Conclusions: Data from our real-world study confirm that VTE is a quite common finding in patients admitted to Internal Medicine departments, and recommended tromboprophylaxis is still underused, in particular in some patients groups. Further efforts are needed to better define the risk profile and to optimize prophylaxis in the heterogeneous setting of medical patients. (c) 2010 Elsevier Srl. All rights reserved.

\section{Introduzione}

Le complicanze tromboemboliche venose (TEV) rimangono un problema di particolare rilievo soprattutto nell'ambito del paziente ricoverato per patologie internistiche acute [1,2] e una delle maggiori cause prevenibili di morbilità e mortalità intraospedaliera. A questo elevato "clinical need" corrispondono, peraltro, evidenze tuttora poco ben definite dal punto di vista sia epidemiologico sia della stratificazione del rischio, così come riguardo alla strategia profilattica ottimale $[3,4]$. Fra le possibili spiegazioni di tale incongruenza vi è la difficoltà di raccogliere informazioni sistematiche in una popolazione eterogenea e complessa quale quella internistica, frequentemente polipatologica e caratterizzata dalla presenza di multipli fattori di rischio paziente-specifici, oltre che da periodi di esposizione al rischio tromboembolico molto meno prevedibili rispetto al paziente postchirurgico $[5,6]$.

Un ulteriore limite è rappresentato dal fatto che buona parte delle informazioni sul problema del livello di rischio di TEV in ambito internistico deriva da studi clinici di intervento farmacologico [7-10]. Questi trial rivestono un particolare significato perché hanno permesso di documentare l'opportunità di utilizzare la profilassi antitrombotica in alcune categorie di pazienti internistici, tuttavia la loro trasferibilità alla realtà clinica è condizionata dai rigidi criteri di selezione della casistica tipici degli studi clinici randomizzati. Inoltre, in questi studi, l'obiettivo primario di valutazione dell'effetto della profilassi è costituito prevalentemente da eventi trombotici asintomatici documentati strumentalmente, la rilevanza clinica dei quali continua a essere oggetto di dibattito fra gli esperti [11].

Lo studio GEMINI, osservazionale e prospettico, condotto in 27 centri aderenti al network FADOI, aveva come obiettivo primario quello di determinare la frequenza di TEV sintomatico correlato all'ospedalizzazione (con sintomatologia comparsa oltre le 48 ore dall'ammissione in ospedale), in una coorte di pazienti consecutivi ricoverati in Medicina Interna. Nel presente articolo vengono riferite, in particolare, le osservazioni raccolte durante lo studio GEMINI relative ai fattori di rischio correlabili all'occorrenza di TEV, nonché quelle riguardanti l'attitudine dell'internista nei confronti dell'impiego della profilassi antitrombotica.

\section{Materiali e metodi}

Lo studio GEMINI è stato condotto durante il periodo marzosettembre 2006 in 27 Unità Operative di Medicina Interna, con il coordinamento scientifico del Centro Studi FADOI. Sono stati arruolati pazienti consecutivi di età $\geq 18$ anni, ricoverati per ogni causa nei centri partecipanti alla ricerca.

Obiettivo primario dello studio era valutare la frequenza di TEV sintomatico "correlato all'ospedalizzazione" (con insorgenza dei sintomi dopo almeno 48 ore dal ricovero). Sulla base di questo obiettivo era stato calcolato un sample size di 4.500 pazienti, idoneo per ottenere una stima affidabile ipotizzando un'incidenza di $0,75 \pm 0,25 \%$ [3]. Più in generale, con lo studio si intendeva documentare, in un contesto real-life, la frequenza complessiva del TEV sintomatico in una popolazione non selezionata di pazienti ricoverati in Medicina Interna, nonché l'impatto clinico di potenziali fattori di rischio per TEV e il comportamento medico rispetto all'utilizzo della profilassi antitrombotica.

Al momento del ricovero venivano raccolte le informazioni salienti riguardanti le caratteristiche del paziente (anamnesi, diagnosi principale al ricovero e patologie concomitanti attive, eventuale presenza di fattori di rischio per TEV, eventuale TEV in atto e relativi dettagli). Alla dimissione venivano invece registrati la possibile insorgenza di TEV "correlato all'ospedalizzazione", l'eventuale ricorso alla profilassi antitrombotica, altri trattamenti effettuati durante il ricovero e l'outcome del paziente.

Lo studio è stato condotto previa approvazione da parte dei Comitati Etici di tutti i centri partecipanti e con l'acquisizione del consenso informato di ciascun paziente osservato nello studio, per il trattamento dei dati personali. La raccolta e la gestione dei dati sono state curate da QBGroup SpA (Padova) attraverso un sistema web-based. Ulteriori dettagli sul disegno e i risultati dello studio sono riferiti in una recente pubblicazione scientifica [12].

\section{Analisi statistica}

Per tutte le variabili di studio sono state utilizzate statistiche descrittive. Per le variabili continue sono stati calcolati indicatori di distribuzione quali media, deviazione standard (DS), mediana, valore minimo e massimo.

L'associazione fra il rilievo di TEV (al ricovero o durante l'ospedalizzazione) e alcuni possibili fattori di rischio è stata valutata mediante analisi multivariata. Le covariate per la regressione logistica sono state selezionate sulla base della letteratura disponibile e della plausibilità clinica; la lista delle variabili comprendeva età ( $>75$ vs $\leq 75$ anni), anamnesi positiva per TEV, recente chirurgia maggiore ( $<3$ mesi), obesità (Body Mass Index $\geq 30$ ), scompenso cardiaco congestizio, broncopneumopatia cronica ostruttiva, neoplasia, 
emi-(para)paresi o emi-(para)plegia, febbre di origine infettiva, allettamento (cronico o $>3$ giorni nelle 4 settimane precedenti l'arruolamento nello studio o $>3$ giorni durante il ricovero). Alcuni possibili fattori prognostici, tra cui trombofilia nota, storia clinica per infarto del miocardio e malattia infiammatoria intestinale, non sono stati considerati nell'analisi perché scarsamente rappresentati nella popolazione in studio o rappresentati con un numero di eventi talmente basso da indurre la presenza di intervalli di confidenza delle stime di correlazione eccessivamente ampi.

Una seconda analisi multivariata è stata condotta per correlare gli stessi fattori prognostici sopra citati con l'eventuale impiego di profilassi antitrombotica.

È stato altresi valutato in quale misura l'impiego della profilassi risultasse coerente con le raccomandazioni espresse

Tabella 1 Caratteristiche generali dei pazienti.

\begin{tabular}{|c|c|}
\hline \multicolumn{2}{|l|}{ Caratteristiche } \\
\hline \multicolumn{2}{|l|}{ Sesso } \\
\hline Donne/Uomini (\%) & $54,6 / 45,4$ \\
\hline \multicolumn{2}{|l|}{ Età } \\
\hline Media \pm DS & $71,0 \pm 15,9$ \\
\hline$\leq 75$ anni (\%) & 51,9 \\
\hline $76-90$ anni (\%) & 41,9 \\
\hline$>90$ anni (\%) & 6,2 \\
\hline \multicolumn{2}{|l|}{ Body Mass Index } \\
\hline$<25,0(\%)$ & 48,1 \\
\hline $25,0-29,9(\%)$ & 33,2 \\
\hline$\geq 30(\%)$ & 18,7 \\
\hline \multicolumn{2}{|l|}{ Clearance della creatinina } \\
\hline$<30 ; \mathrm{L} / \min (\%)$ & 15,1 \\
\hline$<30 \mathrm{~mL} / \min (\%)$ & 24,3 \\
\hline$>50 \mathrm{~mL} / \mathrm{min}(\%)$ & 60,6 \\
\hline \multicolumn{2}{|l|}{ Principali patologie al ricovero (\%) } \\
\hline Diabete & 22,3 \\
\hline Malattia cerebrovascolare & 19,6 \\
\hline Scompenso cardiaco congestizio & 16,5 \\
\hline Neoplasia & 16,4 \\
\hline BPCO riacutizzata & 16,3 \\
\hline Insufficienza renale moderata-severa & 8,1 \\
\hline Arteriopatia periferica & 6,9 \\
\hline Insufficienza epatica lieve-moderata & 6,8 \\
\hline Demenza & 6,0 \\
\hline Infarto del miocardio & 3,0 \\
\hline Malattia ulcerosa & 2,7 \\
\hline Connettivopatia & 1,5 \\
\hline Malattia infiammatoria intestinale & 0,8 \\
\hline \multicolumn{2}{|l|}{ Comorbilità attive al ricovero (\%) } \\
\hline 2 & 30,4 \\
\hline 3 & 16,0 \\
\hline$>3$ & 8,6 \\
\hline \multicolumn{2}{|l|}{ Altre caratteristiche $(\%)$} \\
\hline Allettamento cronico & 8,9 \\
\hline Precedente TEV & 2,5 \\
\hline Recente chirurgia maggiore & 0,9 \\
\hline Trombofilia nota & 0,1 \\
\hline \multicolumn{2}{|l|}{ Durata degenza in Medicina Interna (giorni) } \\
\hline Media \pm DS & $10,9 \pm 10,7$ \\
\hline
\end{tabular}

DS = deviazione standard; BPCO = broncopneumopatia cronica ostruttiva; TEV = tromboembolismo venoso. dalle linee guida redatte nel 2004 dall'American College of Chest Physicians (ACCP) [13], le più recenti disponibili al momento della conduzione dello studio. In questo caso, per definire il paziente "confined to bed" cui si riferiscono le linee guida, è stato selezionato un cut-off di 3 giorni di allettamento sulla base di recenti indicazioni nei pazienti chirurgici [14], dei criteri di inclusione di uno fra i principali trial di tromboprofilassi nel paziente medico [10] e della selezione dei fattori di rischio in una precedente analisi riguardante il TEV nei pazienti ospedalizzati in ambito internistico [3].

Alla luce del riscontro che un consistente numero di pazienti con indicazione alla profilassi, secondo le raccomandazioni ACCP, non l'aveva in effetti ricevuta, è stata quindi condotta un'ulteriore analisi multivariata per valutare quali potessero essere le condizioni correlate alla decisione di non effettuare la profilassi. In questo caso le variabili selezionate sono state: età $\leq 75$ anni, allettamento durante il ricovero $\leq$ 3 giorni, assenza di patologie multiple, presenza di controindicazioni alla profilassi farmacologica (conta piastrinica < 50.000 , emottisi/ematemesi, insufficienza epatica, malattia ulcerosa in atto).

I risultati delle analisi multivariate sono stati espressi in termini di Odds Ratio (OR) e intervalli di confidenza (IC) al $95 \%$, con valori di probabilità $p$ ottenuti con test a due code (statisticamente significativi se $p \leq 0,05$ ).

Le analisi statistiche sono state effettuate utilizzando il programma SAS ${ }^{\circledR}$ (SAS Institute Inc., Cary, North Carolina, USA, versione 9.1.3).

\section{Risultati}

\section{Caratteristiche generali della casistica ed eventi TEV}

Nello studio GEMINI sono stati complessivamente arruolati 4.846 pazienti, la gran parte dei quali di provenienza domiciliare; il 15,2\% dei pazienti risultava invece trasferito in Medicina Interna da altri reparti di degenza ospedaliera. Nella tabella 1 sono descritte le caratteristiche generali della popolazione in studio. Nella tabella 2 sono invece

Tabella 2 Complicanze tromboemboliche venose nei pazienti dello studio GEMINI.

\begin{tabular}{lr}
\hline Tromboembolismo venoso & N. pazienti (\%) \\
\hline Diagnosi prericovero & $31(0,64)$ \\
Diagnosi all'atto del ricovero & $105(2,16)$ \\
TEV con insorgenza sintomi & $15(0,31)$ \\
$\quad<48$ ore dal ricovero & \\
TEV con insorgenza sintomi & $26(0,54)$ \\
$\geq 48$ ore dal ricovero & \\
TEV totale & $177(3,65)$ \\
TVP isolata & 114 \\
Embolia polmonare & 48 \\
TVP + embolia polmonare & 15 \\
TVP arto inferiore & 124 \\
$\quad$ Prossimale & 103 \\
$\quad$ Distale & 21
\end{tabular}

TEV = tromboembolismo venoso; TVP= trombosi venosa profonda. 


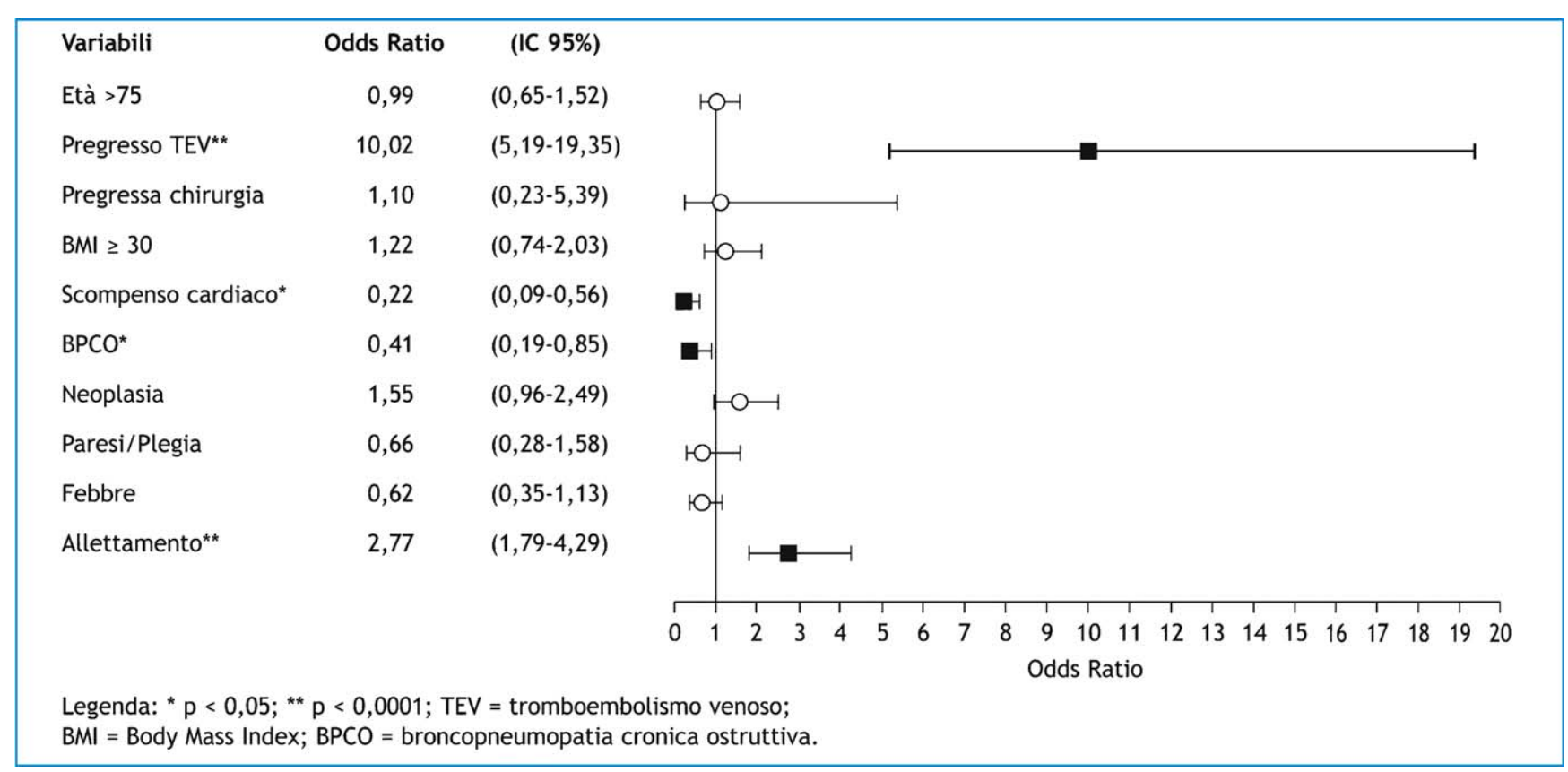

Figura 1 Possibili fattori di rischio e correlazione con l'occorrenza di TEV: analisi multivariata.

riportati i rilievi dello studio in termini di occorrenza di TEV. Nel gruppo di pazienti con TEV la mortalità intraospedaliera è risultata del $5,1 \%$, mentre la mortalità totale durante la degenza, nella popolazione complessiva dello studio, è stata nell'ordine del 6,1\%. Fra i pazienti con TEV correlato all'ospedalizzazione, a un follow-up a 3 mesi non è stata osservata l'occorrenza di recidive; è stata invece rilevata una mortalità per tutte le cause in oltre un quarto dei casi $(26,9 \%)$.

\section{Fattori di rischio per TEV}

I risultati dell'analisi multivariata (Fig. 1) documentano come l'anamnesi positiva per TEV e l'allettamento rappresentino

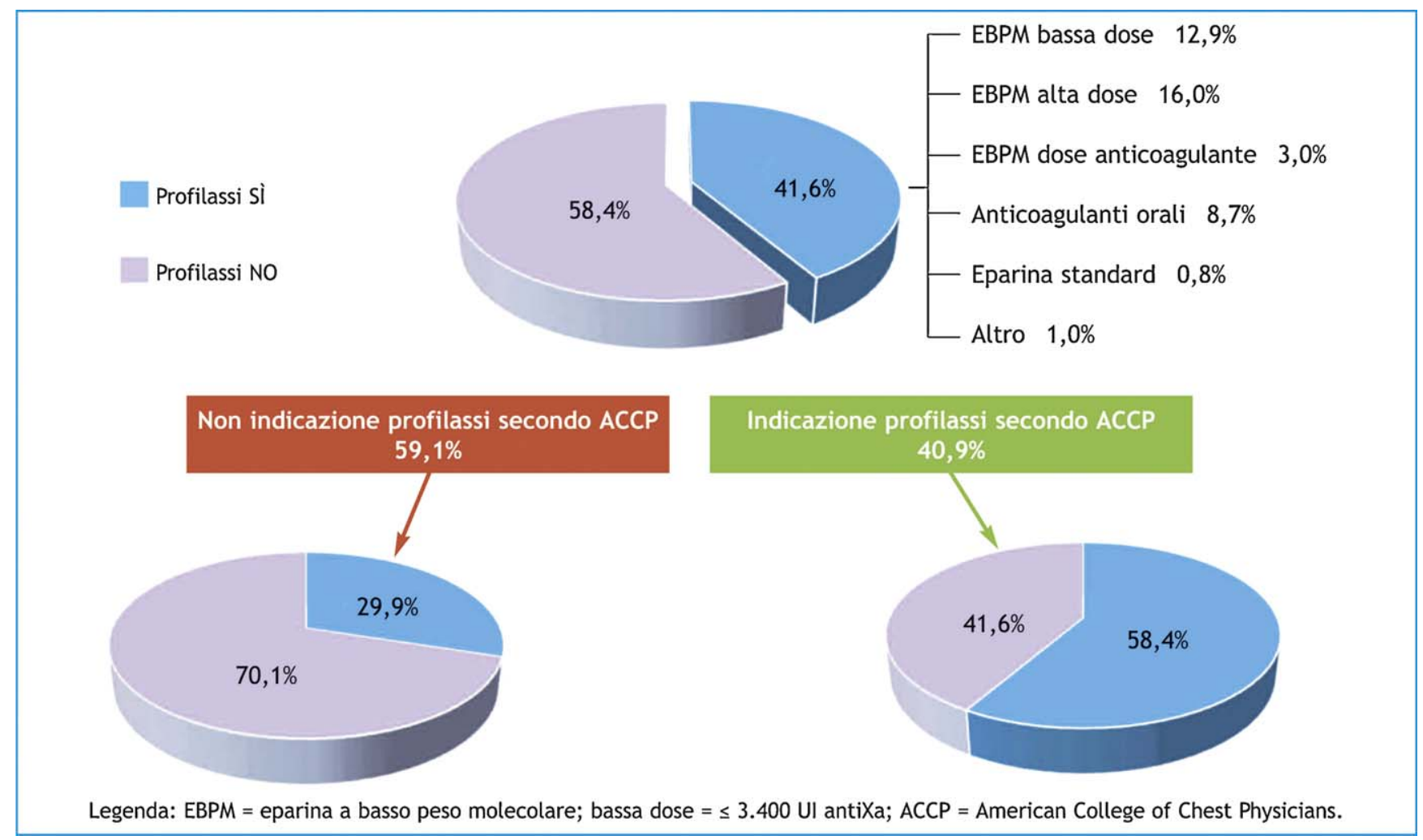

Figura 2 Profilassi per il TEV nei pazienti dello studio GEMINI. 


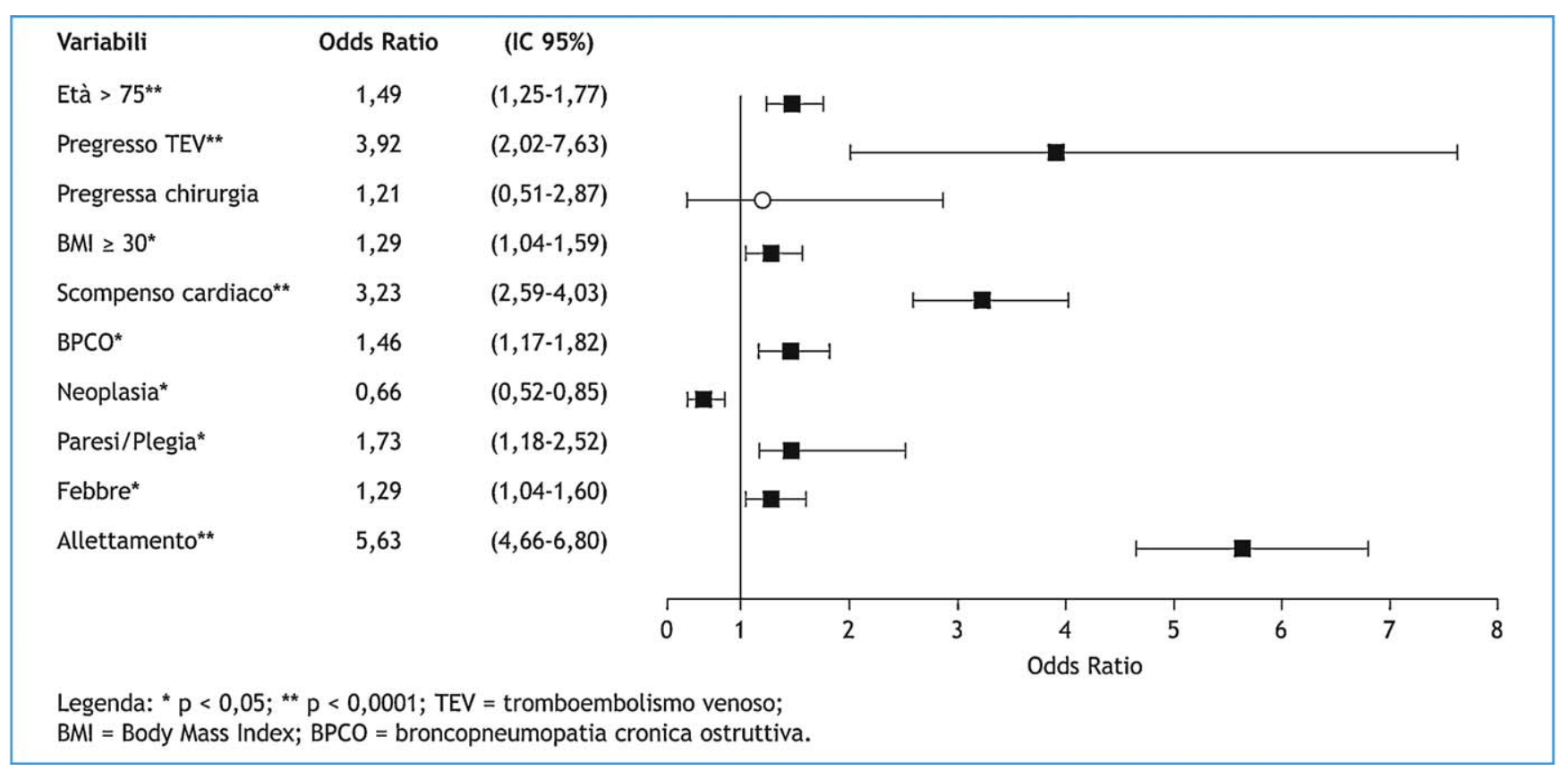

Figura 3 Analisi multivariata per valutare la correlazione tra i fattori di rischio per TEV e l'utilizzo di profilassi antitrombotica in fase di ricovero ospedaliero.

i fattori maggiormente correlati al rischio di complicanze tromboemboliche venose. Un trend per aumentato rischio, ai limiti della significatività statistica, è stato osservato nei pazienti con neoplasia. La presenza di scompenso cardiaco congestizio o di broncopneumopatia cronica ostruttiva è risultata invece inversamente correlata rispetto all'occorrenza di TEV.

\section{Profilassi antitrombotica}

Durante il ricovero ospedaliero una profilassi per TEV è stata adottata nel $41,6 \%$ dei pazienti, utilizzando in prevalenza un'eparina a basso peso molecolare $(31,9 \%$ dei casi), o anticoagulanti orali (8,7\% dei pazienti) (Fig. 2). La durata media della profilassi è risultata di 11,4 $\pm 10,1$ giorni.

Gli stessi parametri valutati come fattori di rischio per TEV sono stati considerati come possibili predittori indipendenti dell'impiego di profilassi antitrombotica. I risultati della relativa analisi multivariata (Fig. 3) documentano come la maggior parte delle variabili considerate appaia significativamente correlata con l'utilizzo di una profilassi antitrombotica, con l'eccezione della chirurgia maggiore nei 3 mesi precedenti e della neoplasia, che presenta una correlazione inversa con l'impiego di profilassi.

Inoltre, l'atteggiamento del medico nei confronti della profilassi è stato valutato in termini di aderenza alle raccomandazioni ACCP [13]. Il 40,9\% dei pazienti osservati nello studio presentava indicazione alla profilassi e, fra essi, il $58,4 \%$ risultava effettivamente trattato durante il ricovero. Una durata dell'allettamento $\leq 3$ giorni (OR 3,58; IC 95\% $3,02-4,68)$, la presenza di una controindicazione alla profilassi farmacologica (OR 3,63; IC 95\% 2,40-4,58) e l'età $\leq 75$ anni (OR 1,34; IC 95\% 1,07-1,67) sono risultate significativamente correlate con la scelta di non effettuare la profilassi nel gruppo di pazienti che pure ne aveva indicazione in accordo alle linee guida ACCP. Fra i pazienti per i quali la profilassi non era raccomandata dalle linee guida ACCP, il $29,9 \%$ ha comunque ricevuto un trattamento antitrombotico (anticoagulanti orali 6,1\% dei casi; eparina 22,4\%; altri metodi $1,4 \%)$.

Alla dimissione, un'estensione della profilassi per il TEV è stata registrata nel $21,1 \%$ dei pazienti (anticoagulanti orali 9,6\%; eparina 9,2\%; antipiastrinici o mezzi fisici 2,3\%).

\section{Discussione}

Solo recentemente la ricerca ha iniziato ad affrontare in maniera più sistematica il problema del TEV nei pazienti con patologie internistiche, ma al momento permangono significative aree di incertezza rispetto alla strategia profilattica ottimale in questo setting clinico. Fra i motivi di tale condizione vi è l'oggettiva difficoltà di studiare un contesto caratterizzato da notevole complessità ed eterogeneità dei pazienti, con periodi di esposizione al rischio difficilmente definibili; occorre inoltre considerare alcuni limiti metodologici derivanti dall'impostazione degli studi disponibili, non sempre sufficientemente rappresentativi della realtà clinica. Da questo punto di vista, studi osservazionali real-life come GEMINI possono proporre informazioni sull'epidemiologia del TEV e sul profilo di rischio nei pazienti internistici in grado di integrare quelle derivanti dai trial di intervento farmacologico, generalmente caratterizzati da criteri selettivi di inclusione dei pazienti.

In prima istanza, lo studio GEMINI conferma che il TEV sintomatico rappresenta una patologia non infrequente nei reparti di Medicina Interna, con una prevalenza complessiva osservata del 3,65\%. Posta in relazione con l'elevato numero di pazienti ospedalizzati in Medicina Interna, questa 
percentuale rende conto del rilevante impatto generale del problema. Nello specifico delle complicanze sintomatiche verosimilmente correlate con l'ospedalizzazione, la percentuale rilevata in GEMINI $(0,55 \%$ dei pazienti senza TEV al ricovero) appare affidabile e verosimile, in quanto coerente con l'ipotesi sperimentale e il dimensionamento dello studio e in linea con altri dati generati in studi osservazionali $[3,15]$ e di intervento $[7,9,10]$.

Come sopra ricordato, uno dei principali problemi nella gestione del TEV in ambito internistico è costituito dalla difficoltà di definire un profilo di rischio del paziente. In letteratura sono stati proposti vari metodi di stratificazione del rischio, come supporto al decision making [16-20], ma essi non dispongono di sufficienti validazioni e risultano comunque scarsamente utilizzati nella pratica clinica. Così pure, rispetto al ruolo di singoli fattori di rischio possibili, i riscontri dei vari studi non infrequentemente risultano in disaccordo fra di loro. Nel nostro studio, un'anamnesi positiva per TEV e l'allettamento prolungato si confermano come condizioni fortemente correlate con il rischio di complicanze tromboemboliche venose. Un riscontro di potenziale rilievo è la persistenza di tale aumentato rischio nonostante nei pazienti con pregresso TEV e in quelli con immobilizzazione o ipomobilità la profilassi fosse utilizzata nella gran parte dei casi. Ciò potrebbe suggerire l'opportunità di considerare, in questi pazienti, l'adozione di misure profilattiche più aggressive rispetto a quanto comunemente fatto.

L'associazione fra tumori e trombosi, nota da molti decenni, ha trovato ampia descrizione in letteratura [21] e si stima che almeno il $20 \%$ dei casi di TEV sia riconducibile alla presenza di una neoplasia [22]. Nello studio GEMINI, ancorché ai limiti della significatività statistica, si è osservata una tendenza a un aumentato rischio di TEV nei pazienti con tumore in fase attiva. Sempre nel nostro studio, peraltro, la presenza di una neoplasia appariva inversamente correlata con l'impiego di profilassi antitrombotica. L'atteggiamento clinico descritto, precedentemente riferito in altre esperienze $[23,24]$, potrebbe essere correlato con l'assenza di precise raccomandazioni per la gestione dei pazienti neoplastici (se non postchirurgici o allettati), con la tendenza a sottovalutare il rischio di una complicanza in presenza di una patologia life-threatening, con i risultati contraddittori di trials di profilassi in categorie specifiche come quella dei soggetti portatori di catetere venoso centrale [25] e/o con la percezione che questi pazienti presentino un rischio di sanguinamento particolarmente elevato. Ciò considerato, i dati dello studio GEMINI sembrano comunque confermare l'esigenza di una migliore caratterizzazione del profilo di rischio in tale eterogenea categoria di pazienti. È altresì possibile che recenti dati che documentano l'efficacia e la feasibility della profilassi farmacologica delle complicanze tromboemboliche in pazienti ambulatoriali in trattamento chemioterapico [26] possano contribuire a rivedere l'atteggiamento terapeutico attuale, o comunque a stimolare l'impegno dei ricercatori in questo settore.

Nello studio GEMINI, in modo almeno apparentemente sorprendente, non è stata evidenziata un'associazione fra rischio di TEV e alcune condizioni quali l'età > 75 anni, la presenza di scompenso cardiaco congestizio, di broncopneumopatia cronica, o di emiparesi-emiplegia. In primo luogo occorre considerare che questi risultati sono stati ottenuti nel contesto di uno studio osservazionale, nel quale l'impiego della profilassi antitrombotica è stato particolarmente esteso soprattutto nei pazienti più anziani e in quelli con scompenso cardiaco, broncopneumopatia cronica o ipomobilità, e ciò può ragionevolmente aver ridotto il rischio di TEV. Inoltre, in termini più specifici, se è vero che l'età avanzata è generalmente considerata un fattore di rischio per TEV [27], e questa correlazione è stata documentata in letteratura [28], parimenti esistono dati che rendono controverso il concetto di età come fattore di rischio indipendente per TEV $[3,29,30]$. Nei pazienti ospedalizzati con scompenso cardiaco o con broncopneumopatia cronica le linee guida ACCP raccomandano l'impiego di una profilassi antitrombotica [1]. In rapporto a tale indicazione sono tuttavia presenti in letteratura dati discordi sul profilo di rischio dei pazienti [28,30-32] e un elemento di particolare rilievo è rappresentato dal fatto che le raccomandazioni ACCP sono fondamentalmente basate sui risultati di trial che hanno selezionato pazienti con scompenso cardiaco [10] o bronconeumopatia cronica [8] a elevato grado di severità. Da questo punto di vista, la popolazione dello studio GEMINI si caratterizzava per una maggiore eterogeneità in termini di severità della patologia. Inoltre, tenuto conto che buona parte degli eventi TEV registrati in GEMINI erano occorsi in fase prericovero, è verosimile che lo scompenso cardiaco e la broncopneumopatia fossero in quella fase presenti, nei pazienti cronicamente affetti, a un basso livello di attività.

Come sopra ricordato, complessivamente il 41,6\% dei pazienti osservati nello studio GEMINI aveva ricevuto un trattamento profilattico nei confronti delle complicanze tromboemboliche venose. Tale percentuale è simile a quella rilevata, nei Paesi occidentali, attraverso un'ampia survey internazionale [33] e leggermente superiore a quanto precedentemente stimato per l'Italia [34]. In questa percentuale globale va considerata una quota di pazienti (circa il $10 \%$ ) che risultava in trattamento anticoagulante orale o antipiastrinico cronico per altre indicazioni, ma classificata dagli sperimentatori nella categoria della profilassi per il TEV. In riferimento alle raccomandazioni delle linee guida ACCP, oltre il $40 \%$ dei pazienti osservati in GEMINI e che avrebbe dovuto ricevere una profilassi antitrombotica, risultava invece non trattato. Questo dato, simile ad altri recentemente pubblicati [35] e, oltretutto, forse sottostimato rispetto alla realtà clinica poiché prodotto in centri in qualche misura sensibilizzati attraverso la partecipazione allo studio, sembra confermare una sottoutilizzazione della profilassi nei pazienti internistici. L'elevato numero di pazienti con indicazione alla profilassi e non trattati è solo parzialmente spiegabile dalla presenza di controindicazioni alla profilassi farmacologica (circa l' $8 \%$ dei casi); inoltre, anche in questo tipo di pazienti sarebbe stato eventualmente possibile il ricorso a profilassi con mezzi fisici, evenienza occorsa, invece, in un numero molto limitato di casi.

Esistono molte possibili spiegazioni alla difficoltà di applicazione della profilassi antitrombotica nei pazienti medici. In primo luogo i pazienti internistici si caratterizzano sovente per la presenza di polipatologie, e il medico può essere maggiormente focalizzato sul trattamento delle malattie in atto piuttosto che sulla prevenzione di una possibile complicanza. La complessità e l'eterogeneità dei pazienti medici rendono inoltre difficoltosa la definizione e la 
validazione di strategie di decision making applicabili in modo sistematico. Non va trascurato il timore del medico di incorrere in complicanze emorragiche correlate al trattamento farmacologico, in pazienti con patologie e trattamenti multipli, aspetto al quale va probabilmente a sommarsi una scarsa fiducia nell'efficacia dei metodi fisici di profilassi $[28,36,37]$.

Infine, come confermato dallo studio GEMINI, fra i pazienti medici è rilevante il peso di alcune categorie, come quella dei soggetti obesi o con insufficienza renale, sovente escluse dagli studi di intervento e per le quali permangono indicazioni non definite rispetto alla strategia profilattica ottimale [38-41]. I dati derivanti dallo studio GEMINI hanno indicato una tendenza a un maggior uso della profilassi nei pazienti con obesità, senza peraltro sostanziali differenze di dosaggio rispetto ai pazienti normopeso. Non è stata osservata una diversa attitudine nella scelta del tipo di profilassi nel caso dei pazienti con insufficienza renale severa, poiché anche in questa categoria di pazienti le eparine a baso peso molecolare hanno rappresentato il presidio di gran lunga più utilizzato, né un'evidente tendenza al ricorso a dosi ridotte di eparina nei pazienti con più severa compromissione della funzione renale. All'epoca dello studio non era commercialmente disponibile la nuova formulazione dell'antitrombotico fondaparinux (1,5 mg/die), specifica per la prevenzione del TEV nei pazienti con clearance della creatinina compresa tra 20 e $50 \mathrm{~mL} / \mathrm{min}$.

L'appropriatezza d'impiego della profilassi nei pazienti medici rimane dunque un "major issue" per gli internisti, che necessita di un importante sforzo di approfondimento. Se da un lato, come documentato da GEMINI, esiste una buona corrispondenza fra il riconoscimento dei principali fattori di rischio per TEV e l'utilizzo della profilassi (con l'eccezione del paziente neoplastico), residua una quota non indifferente di pazienti che non ricevono trattamenti preventivi nonostante per essi le principali linee guida internazionali forniscano specifiche raccomandazioni. Specularmente, nella popolazione di GEMINI, quasi il 30\% dei soggetti che non avevano indicazione alla profilassi hanno invece ricevuto un trattamento preventivo, sulla base di una soggettiva valutazione del rischio da parte del medico curante. Una più appropriata definizione del profilo di rischio dei pazienti, e quindi dell'eventuale strategia profilattica da adottare, appare quindi un'importante esigenza conoscitiva e clinica.

In proposito, può costituire uno spunto di riflessione il rilievo secondo il quale $2 / 3$ dei pazienti che hanno presentato un evento tromboembolico correlato all'ospedalizzazione non avevano ricevuto nessun tipo di profilassi. Per il proprio disegno sperimentale GEMINI non è in grado di fornire indicazioni probanti al riguardo, ma non si può escludere che un utilizzo più ampio della profilassi avrebbe potuto prevenire almeno alcuni di questi eventi. Dall'altro lato, in 9/26 pazienti l'evento tromboembolico correlato all'ospedalizzazione si è manifestato nonostante l'uso di una profilassi. Anche in questo caso le nostre considerazioni sono subordinate al disegno osservazionale dello studio GEMINI, ma è possibile che l'impiego di basse dosi di eparina (in 5 di questi 9 pazienti) possa aver contribuito al failure della profilassi farmacologica. Quello dell'appropriato schema di utilizzo delle eparine è un ulteriore elemento di eterogeneità nella gestione dei pazienti medici e a tal riguardo potrebbe risultare utile la disponibilità di antitrombotici in grado di essere somministrati a dosaggi efficaci fissi [10], semplificando così le strategie terapeutiche.

In conclusione, i risultati dello studio GEMINI documentano come, in un contesto di clinical practice, il TEV sintomatico rappresenti una condizione di frequente riscontro nei ricoveri in Medicina Interna (ne risulta affetto circa 1 paziente su 25), con un impatto assistenziale complessivo rilevante. La profilassi antitrombotica appare globalmente sottoutilizzata, soprattutto in alcune categorie di pazienti a rischio e per le quali è raccomandata l'adozione di misure preventive, così come probabilmente esistono margini di ottimizzazione della profilassi in termini posologici, o di integrazione fra $i$ vari metodi disponibili. In questo senso precedenti esperienze hanno riportato incoraggianti risultati derivanti dall'applicazione di programmi educazionali, di implementazione di linee guida, o comunque di sensibilizzazione su questi argomenti [42,43]. Un importante obiettivo, ancorché di non agevole realizzazione stante la complessità dei pazienti internistici, potrebbe essere la definizione e validazione di metodi easy-to-use in grado di caratterizzare il profilo del paziente e di indirizzare l'operato del medico nei confronti della profilassi del TEV. Oltre a ciò, considerato che i pazienti internistici sono in gran parte dei casi affetti da patologie e fattori di rischio cronici, la riduzione dell'impatto clinico del TEV necessita con ogni probabilità di una maggiore attenzione a tale problematica anche nel contesto della Medicina Generale [44,45].

\section{Note salienti in riferimento allo studio GEMINI}

- GEMINI rappresenta il primo studio che ha valutato contemporaneamente, in un'ampia popolazione non selezionata di pazienti internistici e quindi in un contesto real-world, la frequenza del TEV clinicamente rilevante, i fattori di rischio e l'attitudine dell'internista nei confronti della profilassi antitrombotica.

- Il TEV rappresenta una complicanza di non infrequente riscontro in Medicina Interna, essendo diagnosticato in 1 su 25-30 pazienti ricoverati.

- Facendo riferimento alle principali linee guida internazionali, circa il 40\% dei pazienti ricoverati in Medicina Interna presenterebbe indicazione a ricevere una profilassi per il TEV.

- Circa il 40\% dei pazienti con indicazione alla profilassi con TEV non l'ha invece ricevuta. Questo sottoutilizzo, che può essere almeno in parte ascrivibile alla complessità ed eterogeneità dei pazienti in ambito internistico, richiama l'opportunità di sforzi di approfondimento educazionale e di studio.

- Alcune categorie di pazienti, come i malati oncologici o i pazienti con protratto allettamento, sembrano richiedere una particolare attenzione e probabilmente un'ottimizzazione dell'atteggiamento profilattico.

- Il contesto real-world dello studio ha confermato come non sia trascurabile la percentuale di pazienti appartenenti a categorie (esempio obesità o insufficienza renale severa) sovente escluse dai trial di intervento e per le quali permangono indicazioni non definite rispetto alla strategia profilattica ottimale. 


\section{Conflitto di interesse}

Gli autori dichiarano di essere esenti da conflitto di interessi.

\section{Ringraziamenti}

Siamo riconoscenti a Giovanna Magni (QBGroup SpA, Padova) per l'esecuzione delle analisi statistiche; a Salvatore Corrao (Università degli Studi di Palermo) per il contributo alla pianificazione dello studio; a Davide Ghilardi, Irene Zaratti e Mariagrazia Riciputi (FADOI) per il supporto operativo; al personale infermieristico dei centri partecipanti per la preziosa collaborazione; e ai pazienti inclusi nello studio, per la loro disponibilità e partecipazione.

Lo studio è stato sostenuto da un grant di ricerca non condizionato concesso dall'azienda GlaxoSmithKline Italia.

\section{Appendice. Componenti del gruppo di studio GEMINI}

A. Mazzone, F. Capelli (Ospedale di Legnano, MI); I. Iori, M. Silingardi (Ospedale S. Maria Nuova, Reggio Emilia); M. Mattarei, F. Pugliese (Ospedale S. Maria del Carmine, Rovereto, TN); F. Colombo, P. Fraioli (Ospedale Niguarda, Milano); G. Landini, L. Masotti (Ospedale di Cecina, LI); A. Bulfoni, S. De Carli (Ospedale S. Maria della Misericordia, Udine); G.M. Patrassi (Ospedale di Cittadella, PD); M. Grandi (Ospedale di Sassuolo, MO); G. Iosa (Ospedale di Cesenatico, RN); R. Cavaliere, S. Marengo (Ospedale Mauriziano, Torino); A. Fontanella, D. lannuzzo, P. Di Micco (Ospedale Fatebenefratelli, Napoli); R. Poti, F. Parente (Ospedale Vito Fazzi, Lecce); M. Campanini, A. Airoldi (Ospedale Maggiore della Carità, Novara); F. Salvati (Ospedale SS. Immacolata, Guardiagrele, $\mathrm{CH}$ ); S. Sturbini (Ospedale di Senigallia, AN); G. Vescovo, P. Fanton (Ospedale S. Bortolo, Vicenza); G. Lo Pinto, R. Poggio (Ospedale Galliera, Genova); S. Di Rosa, G. Nicolosi (Ospedale Villa Sofia-CTO, Palermo); R. Laureano, G. Panigada (Ospedale SS. Cosimo e Damiana, Pescia, PT); P. Ghiringhelli, B. Nardo (Ospedale Galmarini, Tradate, VA); A. Sacco, G. Dentamaro (Ospedale Madonna delle Grazie, Matera); P. Pauletto, G. Scannapieco (Ospedale Ca' Foncello, Treviso); C. Pedace, L. Ralli (Ospedale S. Donato, Arezzo); F. Pintus, P. Mascia (Ospedale G. Brotzu, Cagliari); R. Fariello (Ospedale di Chiari, BS); D. Caruso, A. Margarita (Ospedale Cardarelli, Napoli); A. D’Avanzo (Ospedale S.G. Moscati, Avellino)

\section{Bibliografia}

[1] Geerts WH, Bergqvist D, Pineo GF, Heit JA, Samama CM, Lassen MR, Colwell CW. Prevention of venous thromboembolism: American College of Chest Physicians Evidence-Based Clinical Practice Guidelines (8th Edition). Chest 2008;133: 381S-453S.

[2] Heit JA, Silverstein MD, Mohr DN, Petterson TM, O’Fallon WM, Melton 3rd LJ. Risk factors for deep vein thrombosis and pulmonary embolism: a population-based case-control study. Arch Intern Med 2000;160(6):809-15.

[3] Zakai NA, Wright J, Cushman M. Risk factors for venous thrombosis in medical inpatients: validation of a thrombosis risk score. J Thromb Haemost 2004;2(12):2156-61.
[4] Millar JA. Selection of medical patients for prophylaxis of venous thromboembolism based on analysis of the benefithazard ratio. Intern Med J 2009;39(9):606-12.

[5] Oger E, Eressolette L, Nonent M, Lacut K, Guias B, Couturaud F, et al. High prevalence of asymptomatic deep vein thromhosis on admission in a medical unit among elderly patients. The TADEUS Project. Thromb Haemost 2002;88:592-7.

[6] Goldhaber SZ, Tapson VF, DVT FREE Steering Committee. A prospective registry of 5,451 patients with ultrasoundconfirmed deep vein thrombosis. Am J Cardiol 2004;93(2): 259-62.

[7] Samama MM, Cohen TA, Darmon J-Y, Desjardins L, Eldor A, Janhon C, et al. A comparison of enoxaparin with placebo for the prevention of venous thromboembolism in acutely ill medical patients. N Engl J Med 1999;341:793-800.

[8] Fraisse F, Holzapfel L, Couland JM, Simonneau G, Bedock B, Feissel $M$, et al. Nadroparin in the prevention of deep vein thrombosis in acute decompensated COPD. Am J Respir Crit Care Med 2000;161:1109-14.

[9] Leizorovicz A, Cohen AT, Turpie AG, Olsson CG, Vaitkus PT, Goldhaber SZ, PREVENT Medical Thromboprophylaxis Study Group. Randomized, placebo-controlled trial of dalteparin for the prevention of venous thromboembolism in acutely ill medical patients. Circulation 2004;110(7):874-9.

[10] Cohen AT, Davidson BL, Gallus AS, Lassen MR, Prins MH, Tomkowski W, et al., ARTEMIS Investigators. Efficacy and safety of fondaparinux for the prevention of venous thromboembolism in older acute medical patients: randomised placeho controlled trial. Br Med J 2006;332:325-9.

[11] Lowe GD, Sandercock PA, Rosendaal FR. Prevention of venous thromboembolism after major orthopaedic surgery: is fondaparinux an advance? Lancet 2003;362(9383):504-5.

[12] Gussoni G, Campanini M, Silingardi M, Scannapieco G, Mazzone A, Magni G, et al., GEMINI Study Group. In-hospital symptomatic venous thromboembolism and antithrombotic prophylaxis in internal medicine. Findings from a multicenter, prospective study. Thromb Haemost 2009;101:893-901.

[13] Geerts WH, Pineo GF, Heit JA, Bergqvist D, Lassen MR, Colwell CW, Ray GC. Prevention of venous thromboembolism. The Seventh ACCP Conference on Antithrombotic and Thrombolytic Therapy. Chest 2004;126:338S-400S.

[14] Agnelli G, Bolis G, Capussotti L, Scarpa RM, Tonelli F, Bonizzoni $E$, et al. A clinical-outcome based prospective study on venous thromboembolism after cancer surgery - The @RISTOS project. Ann Surg 2006;243:89-95.

[15] Schuurman B, den Heijer M, Nijs AM. Thrombosis prophylaxis in hospitalised medical patients: does prophylaxis in all patients make sense? Neth J Med 2000;56(5):171-6.

[16] Arcelus JI, Candocia S, Traverso Cl, Fabrega F, Caprini JA, Hasty $\mathrm{JH}$. Venous thromboembolism prophylaxis and risk assessment in medical patients. Semin Thromb Hemost 1991;17(Suppl 3): 313-8.

[17] Thromboembolic Risk Factors (THRIFT) Consensus Group. Risk of and prophylaxis for venous thromboembolism in hospital patients. BMJ 1992;305(6853):567-74.

[18] Lutz L. Venous thromboembolism in internal medicine: risk assessment and pharmaceutical prophylaxis. Die Med Welt 2002;53:231-4.

[19] Haas SK. Venous thromboembolic risk and its prevention in hospitalized medical patients. Semin Thromb Hemost 2002; 28(6):577-84.

[20] Cohen AT, Alikhan R, Arcelus JJ, Bergmann JF, Haas S, Merli JG, et al. Assessment of venous thromboembolism risk and the benefits of thromboprophylaxis in medical patients. Thromb haemost 2005;94:750-9.

[21] Falanga A. The incidence and risk of venous thromboembolism associated with cancer and nonsurgical cancer treatment. Cancer Invest 2009;27(1):105-15. 
[22] Imherti D, Agnelli G, Ageno W, Moia M, Palareti G, Pistelli G, et al. Clinical characteristics and management of cancerassociated acute venous thromboembolism findings from the MASTER Registry. Haematologica 2008;93:273-8.

[23] Kahn SR, Panju A, Geerts W, GF, Desjardins L, Turpie AG et al. for the CURVE Investigators. Multicenter evaluation of the use of venous thromboembolism prophylaxis in acutely ill medical patients. Thromb Res 2007;119:145-55.

[24] Jubelirer SJ, Reyes B, Boce M, Elhabyan AK, Mimnagh K, Rosencrance JG, et al. Pharmacologic thromboembolic prophylaxis for medical patients in a tertiary care, teaching community hospital: room for improvement. W V Med J 2008;104:10-4.

[25] Young AM, Billingham LJ, Begum G, Kerr DJ, Hughes AI, Rea DW, et al. Warfarin thromboprophylaxis in cancer patients with central venous catheters (W ARP): an open-label randomised trial. Lancet 2009;373:567-74.

[26] Agnelli G, Gussoni G, Bianchini C, Verso M, Mandalà M, Cavanna L, et al., PROTECHT Investigators. Nadroparin for the prevention of thromboembolic events in ambulatory patients with metastatic or locally advanced solid cancer receiving chemotherapy: a randomised, placebo controlled, double-blind study. Lancet Oncol 2009;10(10):943-9.

[27] Silverstein MC, Heit JA, Mohr DN, Petterson TM, O'Fallon WM, Melton III LJ. Trends in the incidence of deep vein thrombosis and pulmonary embolism. Arch Intern Med 1998;158:585-93.

[28] Alikhan R, Cohen AT, Combe S, Samama MM, Desjardins L, Eldor A, et al., MEDENOX Study. Risk factors for venous thromboembolism in hospitalized patients with acute medical illness. Analysis of the MEDENOX Study. Arch Int Med 2004;164:963-8.

[29] Anderson Jr FA, Wheeler HB, Goldberg RJ, Hosmer DW, Pathwardan NA, Jovanovic B, et al. A population-based perspective of the hospital incidence and case-fatality rates of deep vein thrombosis and pulmonary embolism: the Worcester DVT Study. Arch Intern Med 1991;151:933-8.

[30] Weill-Engcrer S, Meaume S, Lahlou A, Piette F, Saint-Jean O, Sachet $A$, et al. Risk factors for deep vein thrombosis in inpatients aged 65 and older a case-control multicenter study. J Am Geriatr Soc 2004;52:1299-304.

[31] Samama MM. An epidemiologic study of risk factors for deep vein thrombosis in medical outpatients: the Sirius study. Arch Intern Med 2000;160(22):3415-20.

[32] Alikhan R, Spyropoulos AC. Epidemiology of venous thromboembolism in cardiorespiratory and infectious disease. Am J Med 2008;121(11):935-42.

[33] Cohen AT, Tapson VF, Bergmann J-F, Goldhaber SZ, Kakkar AK, Deslandes B, et al., ENDORSE Investigators. Venous thromboembolism risk and prophylaxis in the acute hospital care setting (ENDORSE study): a multinational, cross-sectional study. Lancet 2008;371:387-94.
[34] Cohen AT, Agnelli G, Anderson FA, Arcelus JI, Bergqvist D, Brecht JG, et al. Venous thromboembolism VTE in Europe. The number of VTE events and associated morbidity and mortality. Thromb Haemost 2007;98:756-64.

[35] Chopard P, Dorffler-Melly J, Hess U, Wuillemin WA, Hayoz D, Gallino A, et al. Venous thromboembolism prophylaxis in acutely ill medical patients: definite need for improvement. J Intern Med 2005;257:352-7.

[36] Kleber FX, Witt C, Vogel G, Koppenhagen K, Schomaker U, Flosbach CW, THE-PRINCE Study Group. Randomized comparison of enoxaparin with unfractionated heparin for the prevention of venous thromboembolism in medical patients with heart failure or severe respiratory disease. Am Heart J 2003;145(4): 614-21.

[37] Spyropoulos AC. Emerging strategies in the prevention of venous thromboembolism in hospitalized medical patients. Chest 2005;128(2):958-69.

[38] Hirsh J, Raschke R. Heparin and low-molecular-weight heparin: the Seventh ACCP Conference on Antithrombotic and Thrombolytic Therapy. Chest 2004;126(3 Suppl):188-203S.

[39] Mahè I, Aghassarian M, Drouet L, Bal Dit-Sollier C, Lacut K, Heilmann JJ, et al. Tinzaparin and enoxaparin given at prophylactic doses for eight days in medical elderly patients with impaired renal function. A comparative pharmacokinetic study. Thromb Haemost 2007;97:581-6.

[40] Dentali F, Riva N, Gianni M, Malato A, Bozzato S, Bemasconi M, et al. Prevalence of renal failure and use of antithrombotic prophylaxis among medical inpatients at increate risk of venous thromboembolic events. Thromb Res 2008;123:67-71.

[41] Douketis J, Cook D, Meade M, Guyatt G, Geerts W, Skrobik Y, et al. Prophylaxis against deep vein thrombosis in critically ill patients with severe renal insufficiency with the low-molecularweight heparin dalteparin. An assessment of safety and pharmacodynamics: the DIRECT Study. Arch Intern Med 2008;168: 1805-12.

[42] Labarere J, Bosson JL, Brion JP, Fabre M, Imbert B, Carpentier P, Pernod $\mathrm{G}$, et al. Validation of a clinical guideline on prevention of venous thromboembolism in medical inpatients a before-andafter study with systematic ultrasound examination. J Intern Med 2004;256:338-48.

[43] Dobesh PP, Stacy ZA. Effect of a clinical pharmacy education program on improvement in the quantity and quality of venous thromboembolism prophylaxis for medically ill patients. J Manag Care Pharm 2005;11(9):755-62.

[44] Spencer FA, Lessard D, Emery C, Reed G, Goldberg RJ. Venous thromboembolism in the outpatient setting. Arch Intern Med 2007;167(14):1471-5.

[45] Goldhaber SZ. Outpatient venous thromboembolism: a common but often preventable public health threat. Arch Intern Med 2007;167(14):1451-2. 